\title{
A RIESZ PRODUCT PROOF OF THE WIENER-PITT THEOREM
}

\author{
COLIN C. GRAHAM
}

\begin{abstract}
A Riesz product proof of the Wiener-Pitt theorem is given.
\end{abstract}

Let $G$ be a nondiscrete locally compact abelian group and $M(G)$ the algebra of regular Borel measures on $G$. The (abstract generalization of the) theorem of Wiener and Pitt [WP] is:

THEOREM. There exists a measure $\mu$ on $G$ whose Fourier-Stieltjes transform $\hat{\mu}$ is bounded away from 0 on the dual group $\hat{G}$ of $G$, and such that $\mu$ is not invertible in $M(G)$.

Most proofs of this theorem (e.g. [R, p. 107]) use the existence of thin sets and measures (with certain "pathological" properties) concentrated on those thin sets. We use instead the easiest part of Riesz product theory, in the notation of [HZ] (or see [HR, pp. 425-427]); this appears in (D) below.

The proof of the Theorem proceeds in steps; we leave out some of the details. We will reduce to the case that $G$ is compact.

(A) To prove the Theorem it is enough to show that $G$ contains a closed subgroup $H$ for which the Theorem holds.

(B) If the Theorem holds for the circle group $T$, then it holds for the line $R$. Indeed, consider a measure $\mu$ on $T$ as in the Theorem. By replacing $\mu$ with $\mu * \tilde{\mu}$, we may assume $\hat{\mu} \geqq \delta>0$ on $\boldsymbol{Z}$. According to [Go], there is a measure $\mu^{\prime}$ on $R$ whose Fourier-Stieltjes transform extends that of $\mu$ (on $Z$ ) and is linear on the gaps between consecutive integers. Then $\mu^{\prime}$ on $R$ satisfies the Theorem.

(C) It is enough to prove the Theorem for compact groups. Indeed, apply (A), (B), and the structure theorem [R, p. 40] for locally compact abelian groups.

(D) We now assume that $G$ is compact, and that $\Delta=\left\{\chi_{j}\right\}_{1}^{\infty}$ is an infinite dissociate set [HZ], [HR, p. 425] in the dual $\hat{G}$; that is, if $\chi_{1}, \cdots, \chi_{n} \in \Delta$ and $\varepsilon_{1}, \cdots, \varepsilon_{n} \in\{ \pm 1, \pm 2,0\}$ and if $\sum_{1}^{n} \varepsilon_{j} \chi_{j}=0$, then $\varepsilon_{1} \chi_{1}=\cdots=\varepsilon_{n} \chi_{n}=0$.

Received by the editors July 26, 1973.

AMS (MOS) subject classifications (1970). Primary 43A25, 42A72; Secondary 42A44.

(c) American Mathematical Society 1974 
We let $\mu$ be the Riesz product for $\Delta$ which has Fourier-Stieltjes transform constantly $\frac{1}{2}$ on $\Delta$; that is, the Fourier-Stieltjes transform $\hat{\mu}$ of $\mu$ is given by:

$$
\begin{aligned}
\hat{\mu}(\chi) & =1 & & \text { if } \chi=0 \\
& =2^{-n} & & \text { if } \chi= \pm \chi_{j_{1}} \cdots \pm \chi_{j_{n}}, j_{1}<\cdots<j_{n} \\
& =0 & & \text { otherwise. }
\end{aligned}
$$

(E) We claim that $\frac{1}{2}$ is not isolated in the spectrum of $\mu$. Indeed, if $\frac{1}{2}$ were isolated, then by Gel'fand's operational calculus theorem [Ge], there would exist $v \in M(G)$ such that the Gel'fand transform of $v$ is one where the Gel'fand transform of $\mu$ is $\frac{1}{2}$, and zero elsewhere. This implies that the support of the Fourier-Stieltjes transform of $v$ is exactly $\Delta$. But by a theorem of Rudin (cf. [R, p. 128] or [HR, pp. 420-427]), $\Delta$ is a $\Lambda(2)$ set and hence there exists $C>0$ such that $\|\nu * \omega\|_{2} \leqq C\|\nu * \omega\|_{1}$ for every $\omega \in L^{1}(G)$ such that $\hat{\omega}$ has finite support. By using a bounded approximate identity $\left\{\omega_{\alpha}\right\}$ for $L^{1}(G)$, we now see that $\hat{v} \in L^{2}(\hat{G})$, so that $\Delta$, which is $\{\chi \in \hat{G}: \hat{v}(\chi)=1\}$, must be finite. This contradiction shows that $\frac{1}{2}$ is not isolated in the spectrum of $\mu$. Therefore, the spectrum of $\mu$ contains a number $\lambda$ that does not belong to the closure of the range of $\hat{\mu}$. If $\delta$ denotes the identity of $M(G)$, then $\mu-\lambda \delta$ is not invertible in $M(G)$, but its Fourier-Stieltjes transform is bounded away from 0 . This proves the Theorem for $G$ compact.

Remarks. (i) A difficult result in $[\mathrm{HZ}]$ shows in fact that 1 is not isolated in the spectrum of $\mu$, while an elaboration of the present method shows $2^{-n}$ is not isolated in the spectrum of $\mu$ for $n=2,3, \cdots$.

(ii) We are grateful to the referee for several good improvements in the exposition and organization of the paper.

(iii) O. C. McGehee kindly points out that the (finite Riesz) products $f_{n}(x)=\prod_{j=1}^{n}\left(1+(i / \sqrt{ } n)\left(\gamma_{j}, x\right)\right)$ have $\left|\int f_{n} d v\right| \geqq \sqrt{ } n$ and $\left\|f_{n}\right\|_{\infty} \leqq e$, if $\hat{v}$ is the characteristic function of $\Delta$. This simplifies (E).

(iv) This result is contained in a deep study of Riesz products by Gavin Brown, Riesz products and generalized characters, (1973) (preprint).

\section{REFERENCES}

Ge. I. M. Gel'fand, Normierte Ringe, Mat. Sb. 9 (51) (1941), 3-24. MR 3, 51.

Go. R. R. Goldberg, Restrictions of Fourier transforms and extension of Fourier sequences, J. Approximation Theory 3 (1970), 149-155. MR 41 \#5885.

HR. E. Hewitt and K. Ross, Abstract harmonic analysis. Vol. II: Structure and analysis for compact groups analysis on locally compact Abelian groups, Die Grundlehren der math. Wissenschaften, Band 152, Springer-Verlag, Berlin and New York, 1970. MR 41 \#7378. 
HZ. E. Hewitt and H. S. Zuckerman, Singular measures with absolutely continuous convolution squares, Proc. Cambridge Philos. Soc. 62 (1966), 399-420. MR 33 \#1655.

R. W. Rudin, Fourier analysis on groups, Interscience Tracts in Pure and Appl. Math., no. 12, Interscience, New York, 1962. MR 27 \#2808.

WP. N. Wiener and H. R. Pitt, On absolutely convergent Fourier transforms, Duke Math. J. 4 (1938), 420-436.

Department of Mathematics, Northwestern University, Evanston, Illinois 60201 